\title{
EL QUIEBRE ONTOLÓGICO A PARTIR DEL CONTACTO MAPUCHE HISPANO
}

\author{
José Quidel Lincoleo ${ }^{1}$
}

\section{Introducción o Preámbulo}

Agradezco la invitación, por ende la posibilidad de comentar y ayudar a pensar desde nuestras perspectivas a los escritos del señor Tom Dillehay.

Para leer su trabajo me situaré desde la diferenciación y llevado este a niveles de quiebre ontológica que el mismo autor menciona en su escrito.

Las rupturas ontológicas entendidas como una manifestación profunda de la alteridad humana y sobre todo dado en el contexto hispano mapuche. El pensar, hacer y mostrarse desde sus propias construcciones y de cómo se defiende y proyecta esta ruptura no solo en las formas de vida, sino también en la organización sociopolítica y la forma de ordenarse geopolíticamente.

Mi intención es hacer uso de nuestra perspectiva como mapuche, visibilizar las categorías propias para comentar el escrito. Es este un trabajo que venimos desarrollando desde hace un buen tiempo diversos actores del mapuche rakizuam (pensamiento mapuche) como un ejercicio de descolonización y reconstrucción de nuestras formas de hacer rakizuam (pensar) y ejercer nuestra soberanía epistémica.

La ruptura ontológica del que habla Dillehay posee una gran importancia y es un tema clave para entender las grandes distancias que se manifiestan en aquellos encuentros y que aún en la actualidad siguen penando.

A partir de la ruptura ontológica poder terminar el aniquilamiento comenzado desde la Colonia es quizás la apuesta de los poderes neocoloniales del presente. Más allá de los Estados, desde la avanzada elite económica hegemónica del mundo, hoy los pueblos indígenas de América enfrentan nuevos desafíos, ya no es el hambre, el fusil, sino la vieja estrategia ideológica evangelizadora, pero en la actualidad desde una perspectiva mucho más radicalizada y virulenta, confrontacional, desautorizadora y desde luego, demonizadora perpetrada desde las corrientes más conservadoras como el pentecostalismo.

\section{La Prolongación de la Colonia}

Cuando el autor plantea que en la actualidad no es sostenible los mundos que los europeos crearon en el siglo XVI, "paganos, bárbaros, y los idólatras", para definir a los "otros" pueblos, nosotros sostenemos que estas improntas coloniales continúan operando en gran parte del imaginario sociopolítico chileno.

Al decir de Muyolema (2001), “...los conquistadores instalan un punto de vista perdurable, transhistórico. Toma de posesión del espacio y desplazamiento de la palabra fueron actos simultáneos de despojo sistemático". Por ello, hoy estamos empeñados en re-instalar nuestras palabras para volver a entendernos y hacer entender a los y las "otra/o" en el campo del diálogo escrito.

Nos cabe aquí proponer varias interrogantes que situarán el escrito como una abertura de posibles métodos diferentes de repensar la historia. ¿Cómo reinstalamos una historia que dialogue? ¿Cómo salimos de esta monohistoria de narrativas coloniales, hegemónicas y estandarizadora de categorías exógenas para ver y leer la diversidad? El nombrar, entiéndase categorizar, conceptualizar, es un acto de poder y que enmarca, muchas veces condena a un pueblo a sitiales de marginalidad y penumbra permanente. Muyolema señala que la historia occidental pasa por el uso de sus lenguajes y supuso siempre una política del nombrar, invisibilizando con ello las otras formas de nombrar.

La disputa de la soberanía epistémica es hoy más válido que nunca y se hace necesario plantearlo de manera categórica para rearmar nuestros horizontes simbólicos que cada vez se ven con mayor dificultad. El volver a usar nuestras formas de nombrar, categorizar, estructurar es poner de manifiesto la

1 Centro de Estudios e Investigaciones Mapuche, Comunidad de Historia Mapuche, Temuco, Chile. quidelin@gmail.com 
existencia y vigencia de nuestro mapuche rakizuam (pensamiento mapuche). En este sentido, queremos hacer uso de la antropología como disciplina para enunciar y hacer una antropología desde otras bases epistémicas que logren abarcar las siempre "otras" dimensiones y formas de construcción y objetivación del mundo.

Todo ello para extender un campo disciplinario que denuncie la colonialidad extendida que continúa operando en la forma de nombrar, enunciar, categorizar o intentar imponer categorías y construcciones desde un pensar hegemónico.

Hablamos de prolongación de la colonia siguiendo al propio Dillehay que en su texto expone: "Sin embargo, muchos pueblos indígenas y pobres del mundo están siendo vistos como hacia atrás, sin desarrollo, e indigno o incapaz de autogobierno y autodeterminación; ellos a menudo son vistos como un obstáculo y molestia para el desarrollo global. En muchos sentidos, nuestro mundo actual y la actitud hacia las personas indígenas son todavía una extensión y continuación de los del siglo 16" (la traducción del inglés es mía).

El 24 de agosto 1928 en su editorial el diario $E l$ Mercurio titulaba: "Los indígenas de los alrededores de Temuco son una verdadera rémora para el progreso de la ciudad". Esta idea viene siendo propagada desde los albores de dicha prensa. En su contenido señalaba:

Temuco en su 38 años de existencia se ha transformado en una de las ciudades más importantes de la República, pero a medida que va creciendo se le agrava el problema de ser una ciudad sin producción en sus alrededores, porque los terrenos están entregados a manos de indígenas, que los trabajan escasamente dada la naturaleza holgazana, limitan sus esfuerzos al mínimum de incomodidades para poder vivir ( $E l$ Mercurio 1928).

Cuando se hace difícil invisibilizar entonces hay que descalificar, hacer creer al colectivo, al país que existe un estorbo, algo que impide el bienestar de un bien mayor, en este caso al bienestar de una nación. En la actualidad esta misma campaña tiene otro objetivo y otros ribetes, el mapuche pasó de ser flojo, borracho a terrorista. El tenor de estos discursos siempre apuntan a deslegitimar cualquier apuesta política de los mapuche. El "indio" o "india" es bueno o buena mientras sea útil al desarrollo de la elite chilena.

Para los mapuche esta dialéctica colonial, donde algunas y algunos estarían destinados a ser servidos por el "indio", a vivir usufructuando de su fuerza de trabajo, fue reintroducida por la República chilena con la conquista y ocupación del Gülu Mapu en el siglo XIX (Nahuelpan 2015:272)

Si bien al comienzo de la conformación del Estado Nación chileno, había una otra visión del mapuche y se intentó hacer práctica, sin embargo esta no prosperó:

Se percibe un ambiente de profundas buenas intenciones en la construcción del nuevo Estado Nación que se estaba formando, primando la idea de una gran hermandad. En esta dirección habría apuntado, por ejemplo un proyecto de "Pacificación de la Araucanía" presentado en el año 1823 por Mariano Egaña, que permitiese ocupar la región con colonos nacionales y extranjeros, prefiriendo para ello a los propios mapuches (Bengoa 2004:317).

La prolongación de la colonia se vio fuertemente reforzada al ser parte del eje interpretativo barbarie, primitivismo hacia los indígenas versus la civilización a la que se adscribían.

La sociedad chilena, agraria, santiaguina, que miraba hacia Europa y que surgió en las primeras décadas del siglo XIX, no tuvo la capacidad de comprender al Pueblo Mapuche. Así, desde la capital, los araucanos eran mirados con conmiseración: “... Eran seres primitivos, salvajes; a lo más, bárbaros. En esas tierras del sur de Chile no había llegado aún la civilización..." (Informe CVHYNT 2008:355).

Lo anterior nos lleva a pensar lo difícil que significa pensar en términos de autonomía, autogobierno hacia los mapuche; en estos contextos discursivamente se denotan su violencia, descalificación y prepotencia. 


\section{La Trayectoria de la Idea de Estado Como Proyección e Itinerario de la Idea de Organización desde Una Perspectiva No Indígena}

La idea de Estado se manifiesta como la máxima aspiración en el imaginario intelectual político de Occidente. Sin embargo, tal idea no es considerada en el imaginario indígena. Aunque el significado de Estado haya sido muy diferente en esos otros tiempos, en el caso del pueblo mapuche, la organización sociopolítica estaba estructurada y distribuida según las concepciones de territorialidad de la época. Los lof mapu, rewe, epu rewe, küla rewe, meli rewe, kechu rewe o ayja rewe fueron pequeñas y grandes unidades sociopolíticas sobre la cual operaba el az mapu establecido por los ancianos y poderosos de la época. Nunca se propendió hacia una unificación del pueblo, el horizonte de desarrollo sociopolítico estuvo enmarcado en mantener las autonomías de estas grandes colectividades y los wünen gizol logko y gizol logko se empeñaban en esa tarea al buscar alianzas justamente con otros gizol logko, generalmente de otros fütal mapu y que muy adentrado la Colonia lo continúan los übmeh.

Un problema que se suele ver en los escritos es la definición de lof mapu, invisibilizándose la dimensión del lof che, que Febrés (1965) define como "ranchería" o "parcialidad pequeña".

Sin embargo, las dimensiones mayores como los rewe y ayjarewe se han ido visibilizando con mayor fuerza en los últimos escritos:

Volviendo al concepto de ayllarewe, se puede decir que era considerado por los españoles como la unión de grupos locales vecinos: las parcialidades. Esta unión era visible para los españoles, por una parte, porque existían asambleas regulares de ayllarewe, los coyagtun $\mathrm{y}$, por otra, porque el ayllarewe era representado por un cacique principal o gobernador, un apo-ulmen, el cual trataba juto a los otros caciques o ulmen de ayllarewe, los asuntos de paz con los españoles (Zavala 2008:82).

\section{Concepciones Propias}

Para entender esta ruptura ontológica desde la cual estamos analizando, debemos adentrarnos en la forma mapuche de hacer o entender la política y, en ese caso, ¿cómo se piensa el poder desde esta ontología mapuche?, ¿qué se podría entender como soberanía?, o ¿hacia qué tipo de gobierno, gobernanza podríamos pensar?

La estructura y organización política mapuche proviene de su visión espiritual, es decir, del mapuche gijañmawün. En esta dimensión la sociedad mapuche estructura el orden del wепи тари a partir de los pu $\log k o$, es decir, un grupo de logko que mantienen el orden, se reúnen, conversan y determinan el ordenamiento de la vida cósmica y desde luego de la vida terrenal. La idea de logko es interesante, porque no se piensa en una sola figura de logko, sino de muchos y todos diferentes porque representan diferentes formas de hacer güneluwün. Cada logko posee un $a z$ propio, eso es importante porque es como se manifiesta la diversidad humana. Algunos logko representan fuerzas de lucha de weychan, entonces se denominan weychafeke pu logko, otros son los kimke logko aquellos que poseen el don del conocimiento. Palife pu logko, aquellos que interactúan desde el juego del palin, hampülkafe pu logko, aquellos logko viajeros, bawehzugugechi pu logko, aquellos relacionados con la medicina; en fin, podemos enumerar muchos otros.

Debemos destacar la heterogeneidad de la sociedad mapuche incluso en ámbitos específicos, es lo que queremos poner en evidencia al hablar por ejemplo de güneluwün como la capacidad, potencialidad en la que está forjado el che para asumir su vida plena.

Las personas mapuche no todos somos iguales, cada uno tiene su forma de ver, de interpretar la vida, según como sea su pensamiento y su corazón y así será su forma de actuar y de ser. A lo mejor usted se levanta de madrugada y va al chorrillo a gijatukar, y tal vez este otro peñi no lo hace, tal vez el jujatuka con muday o tal vez el otro lo puede hacer con agua o con unas piedras: eso se llama güneluwün. Así somos los mapuche y la propia tierra también esta de ésa forma. En los diferentes espacios hay diferentes Newen y de acuerdo a eso realizarán su gijatun por eso nosotros no tenemos una religión estructurada, como la religión de los wigka, en cada espacio hay newen y esos newenes son diferentes y la relación de esa gente es de acuerdo a ese newen que existe ahí y que de alguna forma 
se da a conocer (Machi Manuel Lincovil, en Pichinao, Huenchulaf y Mellico Informe COTAM 2003).

Esta idea de güneluwün es la que puede asociarse o entenderse como "poder", a la que ciertos che están en condiciones de asumir y por ello padecer. Es también el concepto mapuche que podemos analogarlo a "política".

La utilización de los conceptos propios, tradicionales u ontológicamente propios, nos lleva hacia un dilema mayor en la actualidad y es que nos encontramos con una sociedad mapuche con sus lazos de afinidades y parentesco cada vez más debilitados, con un alto grado de mestizaje que pone en un estado de gran relatividad los planteamientos sociopolíticos de origen. Frente a esta realidad, no es fácil reinstalar u operativizar los conceptos que se sostienen desde una visión precolonial.

A pesar de las teorías y supuestos de la configuración étnica reche-araucano-mapuche, existen "otras" evidencias desde una mirada "otra" o desde nuestra propia mirada. Una de estas "pruebas" son los discursos rituales con los que interactuamos permanentemente en el mundo que se le denomina "tradicional". El mismo autor Tom Dillehay ha expuesto $^{1}$ de manera íntegra un fabuloso texto en estado de küymin, en el que se denotan estructuras de organización de hace mucho tiempo.

Bajo el esquema del quiebre ontológico, el indigenismo, ha ido socavando las estructuras propias, sobrevalorando la estructura organizacional de orden jurídico para el funcionalismo de sus políticas asistencialistas de mendicidad. Por tanto, la estructura sociopolítica propiamente mapuche sigue operando de manera clandestina, invisibilizada. Pero es "la" forma de organización por excelencia que sostiene las grandes ritualidades y estructura de ordenamiento socioespacial como son los lof mapu у rewe mapu. A esta estructura y líderes son los que tienen de blanco los grupos de evangélicos para demonizar y los que la moderna prensa liberal acusa de resabio de involución y los modernos líderes indígenas y pensadores a su vez tildan de esencialistas.

\section{Relación Territorio-Salud-Che y su Imbricación}

El lenguaje arcaico de los rituales, la temporalidad, tiene otras dimensiones, por ello, antiguas formas de pensar, ordenar, decir, se hacen presentes hasta nuestros días mediante los diferentes ritos de sanación, como zatun o en los geykurewen de los machi en los diferentes territorios. Allí vuelven a tener sentido las antiguas toponimias, los wigkul cerros, bewfü ríos, vuelven a dialogar con los diferentes seres que poblamos este pluriverso en esta dimensión del naӥq тари.

En ninguna parte es más importante esta conexión entre gente, tierra, historia, genealogía, espiritualidad y pertenencia a una nueva sociedad que en los campos de ngillatun, los kuel y los rewekuel de Purén y Lumako (Dillehay 2011:45).

Los mecanismos de conexión interespecies que los mapuche desarrollaron dan muestra de una compleja forma de entender la vida, la organización, la espacialidad y el núcleo de esa conexión es la espiritualidad. Los xeg xeg wigkul, por ejemplo, son desde tiempos milenarios reconocidos como tales y su vigencia es absoluta, lo demostraron en el pasado terremoto del 27 de febrero 2010.

En el fütal mapu naüqche, los kuwel son los testimonios de unión, congregación y protocolo entre territorios, espacios y gente. Un modelo de geopolítica mapuche, basado en las alianzas, pero manteniendo sus autonomías y determinaciones emanadas desde el az mapu correspondiente. Az тари que contiene dos grandes implicancias, como una forma particularizada de establecerse en los espacios locales y desde allí entender, valorar, respetar y vivir una vida. Otra, es a su vez esta capacidad abarcadora y totalizadora que posee en todo el mapuche rakizuam (pensamiento mapuche).

La idea de poder desde lo mapuche es algo que no se ha tratado en los escritos. Poder, soberanía, leyes, gobierno son términos que asocian y poseen una vinculación muy prolífera.

Nuestras concepciones no son dogmáticas ni petrificadas milenariamente, como se suele pensar acerca de nuestras formas de pensar ciertos tópicos, es más, nuestras principales concepciones son tremendamente elásticas y contextualizadas a la realidad de los territorios.

Pese a que, a primera vista, se pueda pensar que el Az Mapu consiste en una serie de dogmas inmutables, en realidad no es así. Nos encontramos ante una serie de proposiciones ancestrales genéricas 
que se prestan a diversas interpretaciones y usos según la época y las condiciones históricas, permitiendo abordar de manera dinámica situaciones de crisis, conflictos e incertidumbres en diferentes momentos. Desde esta perspectiva nos referimos al $\mathrm{Az}$ Мари сomo un conjunto de experiencias, conocimientos ancestrales, prescripciones y normas vinculadas con la creencia religiosa y con la cosmovisión, las cuales pueden ser articuladas y secularizadas para la acción social con relativa flexibilidad (Antona Bustos 2014).

Lo más relevante de estas nociones es que operan de manera sistemática en los diferentes territorios $\mathrm{y}$ en las diversas manifestaciones del mapuche mogen (vida mapuche). Una prueba de ello son los mapuche kuxan que se denominan yafkan, que significan la transgresión de la persona a las normas del az mapu. Estas enfermedades son consideradas de alta complejidad entre las familias mapuche, pues deben ser tratadas bajo el protocolo que los newen establecen por medio de los machi e implican la participación de la familia y comunalidad en red para restablecer el equilibrio perdido.

Subjetividad y objetividad forma en que se manifiesta lo intangible.

La relación cotidiana de los mapuche con la naturaleza no es abstracta sino que conlleva un compromiso individual y familiar con los hábitats de referencia que 'obliga' a través del feyentün (creencia o autorregulación), el kimün (conocimiento ancestral) y el günen (control) a cumplir con los rituales de carácter doméstico para que todo vaya bien. La transmisión del conocimiento relativo a las prescripciones y protocolos que se deben seguir se realiza oralmente a partir de una serie de relatos denominados epew (Antona Bustos 2016).

\section{Idea de mapu}

Debemos reiterar y recordar que la idea de mapu no es solo equivalente a tierra como suele ser traducido generalmente. El término тари es multidimensional, pues se puede aplicar a los espacios tangibles y materiales como en el lof (espacio de comunalidad), los rewe (agrupación de familias lof che o lof mapu), wajontu mapu (planeta), waj тари (universo), ka mapu (espacio de los antepasados).

La idea de mostrar lo anterior es hacer vislumbrar las complejidades que encierran las ideas y categorías mapuche relacionados con las ideas de poder, gobierno, soberanía que son muy diferentes a cómo se piensa y por tanto se plasma en las sociedades no mapuche.

Vanessa Lea (2012) siguiendo a Da Matta, plantea la idea de las relaciones de substancia que aduce a las profundas relaciones entre humanos y las otras vidas establecidas en los espacios territoriales.

La idea de relación entre espacios y humanos, también lo encontramos en las últimas investigaciones andinas:

Es así que el poblador andino no trata con la naturaleza, sino con sujetos que interactúan con él y pueden afectar su vida. La relación no es de sujeto a objeto, materia o recurso, como observa la perspectiva occidental, sino de persona a persona. Por tanto, en los Andes la "naturaleza" es como nosotros, porque la Pachamama y los Apu se molestan, comen, toman, bailan, hablan, discuten, sienten hambre (Torres Lezama 2015).

\section{¿Soberanía o interrelación? Anülmapun como rito de acercamiento y filiación mapu-che}

Anülmapun, es un término clave que nos demuestra la intensa relación entre los che y el mapu. Rito de acercamiento, conocimiento, negociación y pago para establecerse como grupo, reyñma (familia), con los geh mapu existentes.

Bajo esta concepción, estamos entendiendo, entender, aprender y conocer el $a z$ que poseen los geh de un mapu determinado y, a partir de ello, poder establecer un vínculo que permita el mogen (vida).

Desde el punto de vista mapuche, habitar un espacio implica conocer las vidas que en este existen, sean grupos humanos, como otras vidas en un sentido amplio, como los geh (dueños guardianes) у pu newen (fuerzas) que están presentes y pueden llegar a materializarse en un perimontu (Pichinao 2015).

Para entender y profundizar acerca de este ritual continuamos 


\begin{abstract}
Antiguamente, cuando una familia se establecía en un territorio, efectuaba una ceremonia denominada anülmapun (echar raíces, asentarse en el lugar) que consistía en un saludo formal a las vidas inmateriales del territorio. Este era considerado el primer protocolo para propiciar una convivencia equilibrada entre las personas y los demás seres del lugar, de modo que les dejen vivir, les acompañen y no afecten negativamente el cotidiano familiar (Pichinao 2015).
\end{abstract}

Concluyendo, podemos decir, que al no existir tácitamente una idea de "propiedad", más bien una idea de convivencia interespecie, no se hace necesario una soberanía de los espacios, más bien un reconocimiento entre ambas partes.
Entender esta mapu rakizuam y extenderlo es hoy un desafío, aunque en términos académicos, han ido emergiendo nuevas formas de entender e identificar este tipo de pensamiento, Arturo Escobar (2012) le denomina ontología relacional en donde se busca desmontar la clásica visión dualista y reemplazándola por una visión multidimensional del territorio. Desde el mapuche rakizuam, es la idea de que kom mapu ta mogeley, xür mogeleyiñ mapu iñchiñ, mapu ñi zuam mogeleyiñ. Toda los espacios son seres vivos, espacios y nosotros los humanos vivimos juntos, gracias a estos espacios nosotros podemos estar vivos.

Por consiguiente las ideas de soberanía se deben releer desde otras perspectivas que sean incluyentes a esta idea de mogen (vida) de las especies y espacio.

Por último, más que definir, hemos intentado abrir un espacio para seguir el debate y así continuar dialogando.

\section{Referencias Citadas}

Antona Bustos, J. 2014. Los Derechos Humanos de los Pueblos Indígenas. El Az Mapu y el Caso Mapuche. Ediciones Universidad Católica de Temuco. Temuco, Chile.

Antona Bustos, J. 2016. Derechos Indígenas, Conflictos Ambientales y Territoriales: El Caso Mapuche. Editorial Irredentos Libros, Grupo de Acción Comunitaria (GAC), Madrid.

Bengoa, J. 2004. La memoria olvidada. Historia de los pueblos indígenas de Chile. Compilación del Informe de la Comisión de Verdad Histórica y Nuevo Trato. Cuadernos Bicentenarios. Santiago.

Dillehay, T.D. 2011. Monumentos, Imperios y Resistencia en los Andes. El Sistema de Gobierno Mapuche y las Narrativas Rituales. Universidad Católica del Norte, Qillqa.

Escobar, A. 2012. Culturas y diferencia: la ontología política del campo de la cultura y desarrollo. Wale'keru. Revista de investigación en cultura y desarrollo, $\mathrm{N}^{\circ} 2$. www.edu-library. $\mathrm{com} / \mathrm{ca} /$ show?id=596.

Febres, A. 1765. Arte de la Lengua del Reyno de Chile. Calle de la Encarnación, Lima.

Lea, V. 2012. Riquezas Intangíveis de Pessoas Partíveis. Os Mebengokre (Kayapó) de Brasil Central. Editorial Universidade São Paulo, São Paulo.

Nahuelpan Moreno, H. 2015. Nos explotaron como animales y ahora quieren que nos levantemos. Vidas despojables y micropolíticas de resistencia mapuche. En Awükan ka Kuxankan Zugu Wajmapu Mew. Violencias Coloniales en Wajmapu, editado por E. Antileo Baeza, L. Cárcamo-Huechante, M. Calfío Montalva, H. Huinca-Piutrin, pp. 271-300. Ediciones Comunidad de Historia Mapuche, Santiago.
Pichinao, J. 2015a. Ontología mapuche y catástrofes telúricas. En Comunidades de América Latina. Perspectivas Etnográficas de Violencia y Territorio desde lo Indígena, editado por L. Gavilán y V. Torres, 181-189. Ceques Editores, Cusco.

Pinto, J. 2003. La Formación del Estado y la Nación, y el Pueblo Mapuche. De la Inclusión a la Exclusión. Dirección de Bibliotecas, Archivos y Museos, Santiago.

Quidel, J. 2005. Pu Mapuche ka pu wigka, chumgechi ñi xokituwün. Las relaciones interétnicas a través de la religión. El caso de los mapuche y no mapuche en Chile. Anthropos 207, "Antropología y Sociedad" Una relación compleja, crítica y problemática. Propuesta de una antropología interactiva, Barcelona.

Quidel, J. 2012. La Idea de "Dios" y "Diablo" en el Discurso Ritual Mapuche. Las Resignificaciones de las Categoría Dios y Diablo entre las Autoridades Socioreligiosas Mapuche del Territorio Wenteche. Disertación de Maestría, Programa de Postgrado en Antropología Social, Instituto de Filosofía y Ciencias Humanas, Universidad Estadual de Campinas, Campinas.

Torres Lezama, V. 2015. La personificación de Apu y Pachamama y la elaboración de una ofrenda colectiva en el sur andino del Perú. En Comunidades de América Latina. Perspectivas etnográficas de violencia y territorio desde lo indígena, editado por L. Gavilán y V. Torres, 191-212. Ceques Editores, Cusco.

Zavala, J.M. 2008. Los Mapuches del Siglo XVIII. Dinámica Interétnica y Estrategias de Resistencia. Editorial Universidad Bolivariana, Colección Estudios Sociales, Santiago. 


\section{Documentos}

Comisión Verdad Histórica y Nuevo Trato con los Pueblos Indígenas 2008. Informe de la Comisión Verdad Histórica y Nuevo Trato con los Pueblos Indígenas. Edición Comisionado Presidencial para Asuntos Indígenas, Santiago.
Pichinao, J., E. Huenchulaf y F. Mellico 2003. Pu Mapuche ñi Gijañmawün Gulu ka Puwel Mapu mew. La forma mapuche de pensar y practicar la religión. Informe Final COTAM, Temuco.

Diario El Mercurio 1928. Archivo personal.

\section{Nota}

1 O sobreexpuesto. No es recurrente obtener estos tipos de recursos y más todavía exponerlo literalmente. Los discursos rituales son manifestaciones orales que poseen una importancia solemne. No es pensable desde nuestra óptica "revelar" estos discursos, pues tienen y contienen contenidos para ciertos contextos y momentos. La sobreexposición de estos textos pueden ser leídos como una transgresión desde la perspectiva mapuche. 
